\title{
A new lens for observing fundus with a noncontact wide-angle viewing system
}

\author{
This article was published in the following Dove Press journal: \\ Clinical Ophthalmology \\ 3 July 2017 \\ Number of times this article has been viewed
}

\author{
Mihori Kita \\ Yukiko Fujii \\ Sachiyo Hama \\ Department of Ophthalmology, \\ National Organization Kyoto Medical \\ Center, Kyoto, Japan
}

\begin{abstract}
Drying of the cornea during surgery, which causes poor visibility of the fundus, is one of the most serious problems when using a noncontact-type wide-angle viewing system. This study used a newly commercialized lens for observing the fundus with a noncontact wide-angle viewing system. The lens used has a very thin meniscus made of polymethyl methacrylate and a large outer diameter. Following the application of viscoelastic material, the lens can be put on the cornea without using a contact lens fixation ring. The larger diameter and lightweight characteristics of the new lens contributed to the overall stabilization. The smooth surface of this lens was able to achieve an intraoperative view of good quality not only during vitrectomy but also during scleral buckling procedures.
\end{abstract}

Keywords: contact lens, observing fundus, vitrectomy, scleral buckling, noncontact wide-angle viewing system

\section{Introduction}

Recently, the use of a wide-angle viewing system has become the global standard for vitrectomy surgery. The noncontact type of system, which consists of a noncontact front lens, reduction lens, and stereoscopic inverter, is especially more popular because of the stability of the fundus image and the ease of manipulation. Simultaneous use of the system combined with a light fiber illumination for fundus visualization is also feasible for scleral buckling procedures for retinal detachment. ${ }^{1-3}$ However, when performing operations using the noncontact type of wide-angle viewing system, continuous hydration of the cornea is necessary in order to achieve a clear view of the fundus.

We designed and previously reported a prototype of an antidrying lens for vitreous surgery in 2013. ${ }^{4}$ This lens has recently become commercially available for observing the fundus when using a noncontact wide-angle viewing system. This study further describes about the lens and its usefulness during vitreous surgeries.

\section{Subjects and methods}

This study was approved by the Ethics Committee of National Hospital Organization Kyoto Medical Center, and all procedures involved adhered to the tenets of the Declaration of Helsinki.

\section{Lens design}

The lens (HOYA Cooperation, Tokyo, Japan) has a 0.26-mm thickness at the center, a clear meniscus made of polymethyl methacrylate, and includes two gates for handling with forceps. In addition to the large outer diameter that measures $13.0 \mathrm{~mm}$, the lens also has a loose curve that measures $8.40 \mathrm{~mm}$ for the back curve radius (Figure 1). 

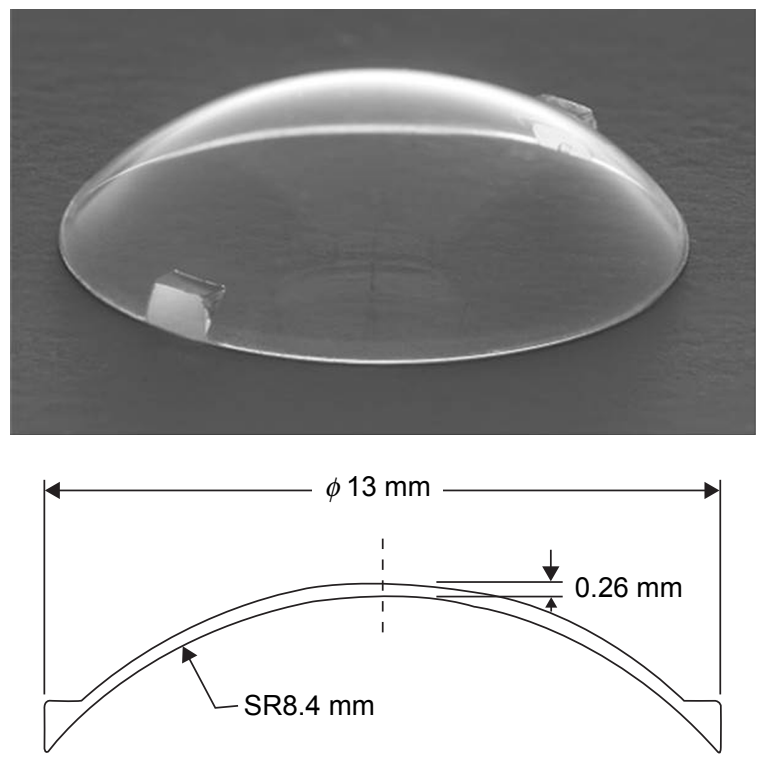

Figure I External view (top) and the schematic diagram of the cross-sectional view of the contact lens (bottom).

Abbreviation: SR, spherical radius.

Fixation rings are not required to hold the lens in place after being applied to the surface. The lens is also designed to have a refractive power of zero and is for single use only.

\section{Subjects}

Between June 2016 and January 2017, the contact lens was used for 82 cases of pars plana vitrectomy (retinal detachment: 51 cases, diabetic retinopathy: 17 cases, vitreous hemorrhage: 7 cases, vitreomacular traction: 5 cases, and vitreous opacity: 2 cases) and 6 cases of scleral buckling with a wide-angle viewing system. All procedures were performed at the Department of Ophthalmology in the National Hospital Organization Kyoto Medical Center. Written informed consent was obtained from all the patients.

\section{Surgical procedures Vitrectomy}

All the surgical cases were performed by a single surgeon (MK) using a 25-gauge vitrectomy system with a wide-angle viewing system $\left(\right.$ Resight $^{\circledR}$, Carl Zeiss Meditec AG, Jena, Germany) or with phacoemulsification and intraocular lens implantation, if necessary. To protect the cornea from drying during the vitreoretinal surgery, viscoelastic material was applied to the corneal surface and then the lens was positioned on the top of the cornea using fingers or a forceps.

\section{Scleral buckling}

Following conjunctival peritomy around the limbus and isolation of the rectus muscles, a 27-gauge light fiber (DORC,
Zuidland, the Netherlands) was placed $4 \mathrm{~mm}$ behind the limbus. A wide-angle viewing system was activated after the contact lens was positioned on the top of the cornea as described previously. The retinopexy procedure was then performed with cryopexy applied to the retinal breaks. The buckling procedure and external drainage of the subretinal fluid were carried out under a microscope. In cases where manipulation and rotation of the eye made the lens surface cloudy with blood, the lens was removed and cleaned. The fundus was checked under a panoramic view using the noncontact wideangle viewing system with the antidrying lens.

\section{Results}

In all cases, the fundus images were more clearly visualized when viewed through the contact lens versus only the viscoelastic material. In the rhegmatogenous retinal detachment or proliferative vitreoretinopathy cases, the retinal breaks at the periphery could be clearly observed through the contact lens during both the fluid and air phases. This improved visibility helped in removing the vitreous traction (Figure 2) and proliferative membrane (Figure 3), in addition to making it possible to safely and effectively perform internal drainage and retinopexy.

The clearer images that could be viewed at the posterior pole through the lens with not only a 128-diopter but also a 60-diopter objective lens made it easier to perform the posterior vitreous separation (Figure 3) and manipulate the proliferative membrane in diabetic retinopathy (Figure 4). In the eyes of the diabetic retinopathy patients who had

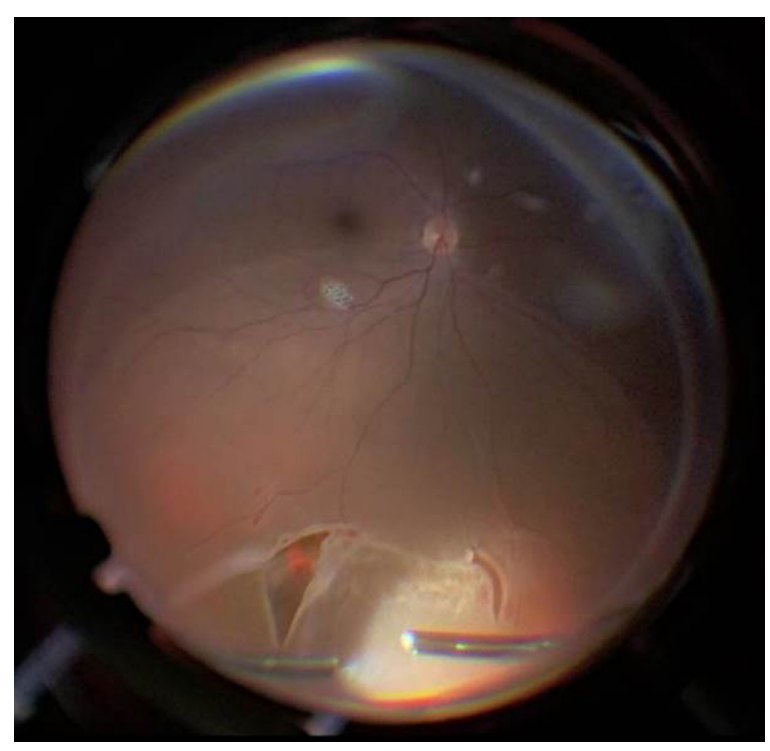

Figure 2 Fundus view of the contact lens with the noncontact wide-angle viewing system in a case of rhegmatogenous retinal detachment. 

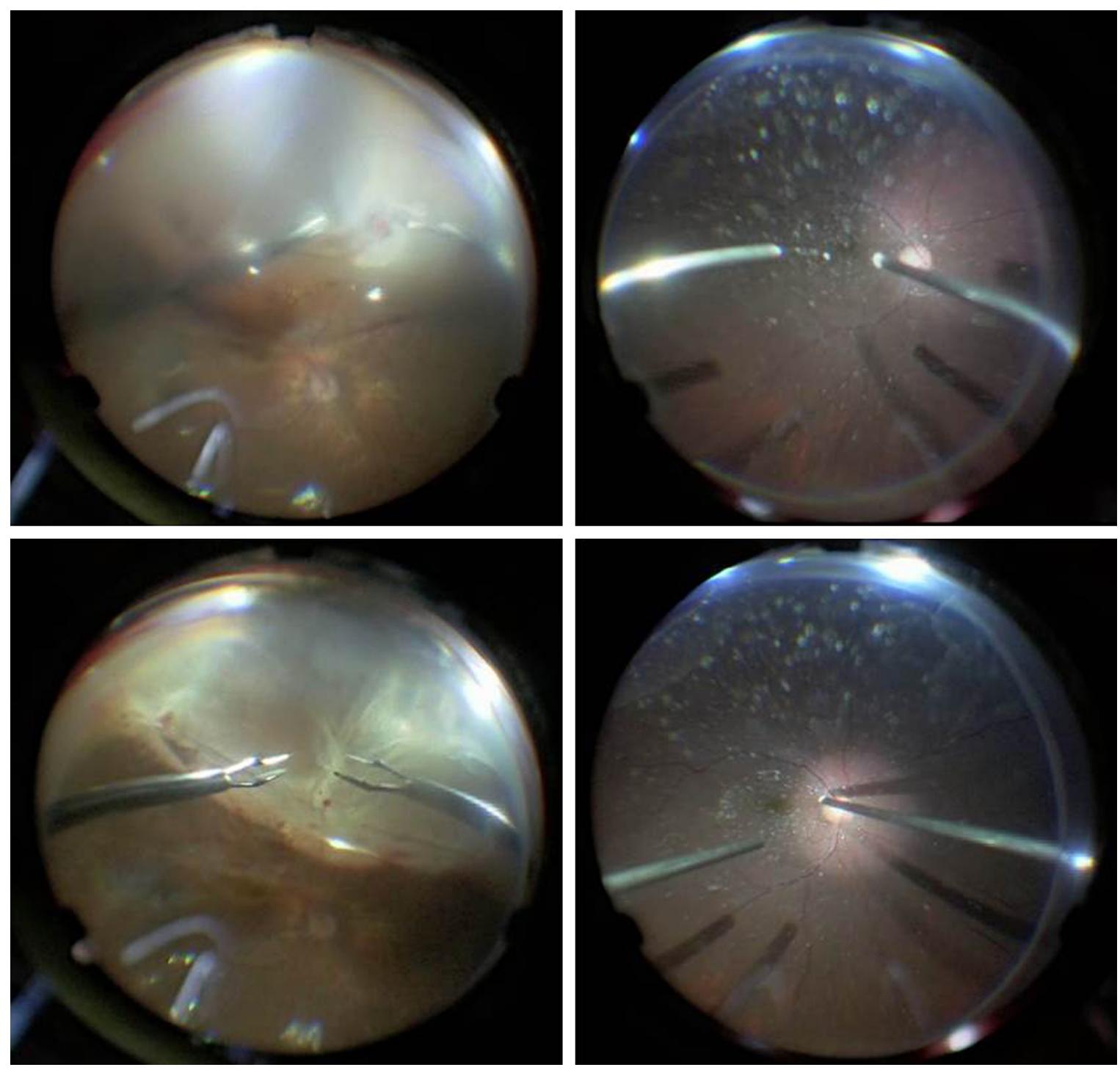

Figure 3 Fundus view of the wide-angle viewing system with a 128-diopter objective lens alone (top), and use of the contact lens with the wide-angle viewing system (bottom) for removal of proliferative membrane in a case of proliferative vitreoretinopathy (left) and for performing posterior vitreous separation in a case of vitreomacular traction (right)

corneal erosion and epithelial edema, use of the antidrying lens instead of epithelial abrasion improved the visualization of the fundus (Figure 5).

Moreover, the use of this lens in scleral buckling procedures also assisted in the observation of the fundus during retinopexy with the wide-angle viewing system and made it possible to perform indirect ophthalmoscopy without continuous hydration by an assistant (Figure 6).

No intra- or postoperative complications were encountered in any of the cases.

\section{Discussion}

Application of a viscoelastic material to the corneal surface has recently become a commonly utilized method for preventing the cornea from drying during surgeries that use a noncontact wideangle viewing system. However, the irregular surface of the viscoelastic material can lead to a poor quality of vision of the surgical field. Thus, the combined use of an antidrying lens and a noncontact wide-angle viewing system appears to be advantageous. The lens not only prevents the cornea from becoming dry but also contributes to a smooth corneal surface, thereby providing a good quality of view of the surgical field.

Previous studies have reported on the usefulness of several types of contact lenses that can overcome drying of the cornea. ${ }^{4-8}$ A magnifying vitrectomy contact lens appears to be the most advantageous, as it can provide a wider fundus view. ${ }^{5,6}$ However, a lens holder or a fixation ring is necessary when using this procedure. While a rigid gas-permeable 

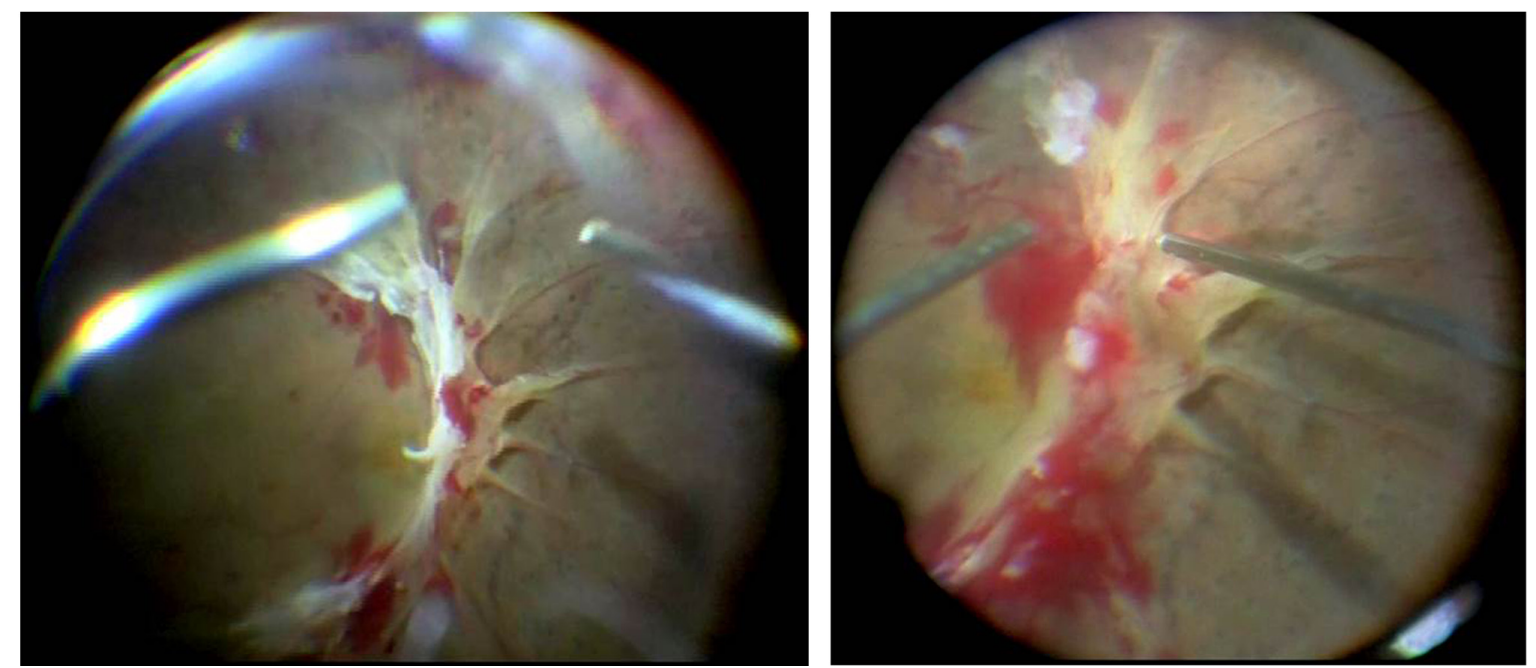

Figure 4 Fundus view through the contact lens with a 128-diopter objective lens (left) and with a 60-diopter objective lens (right) in a case of proliferative diabetic retinopathy.
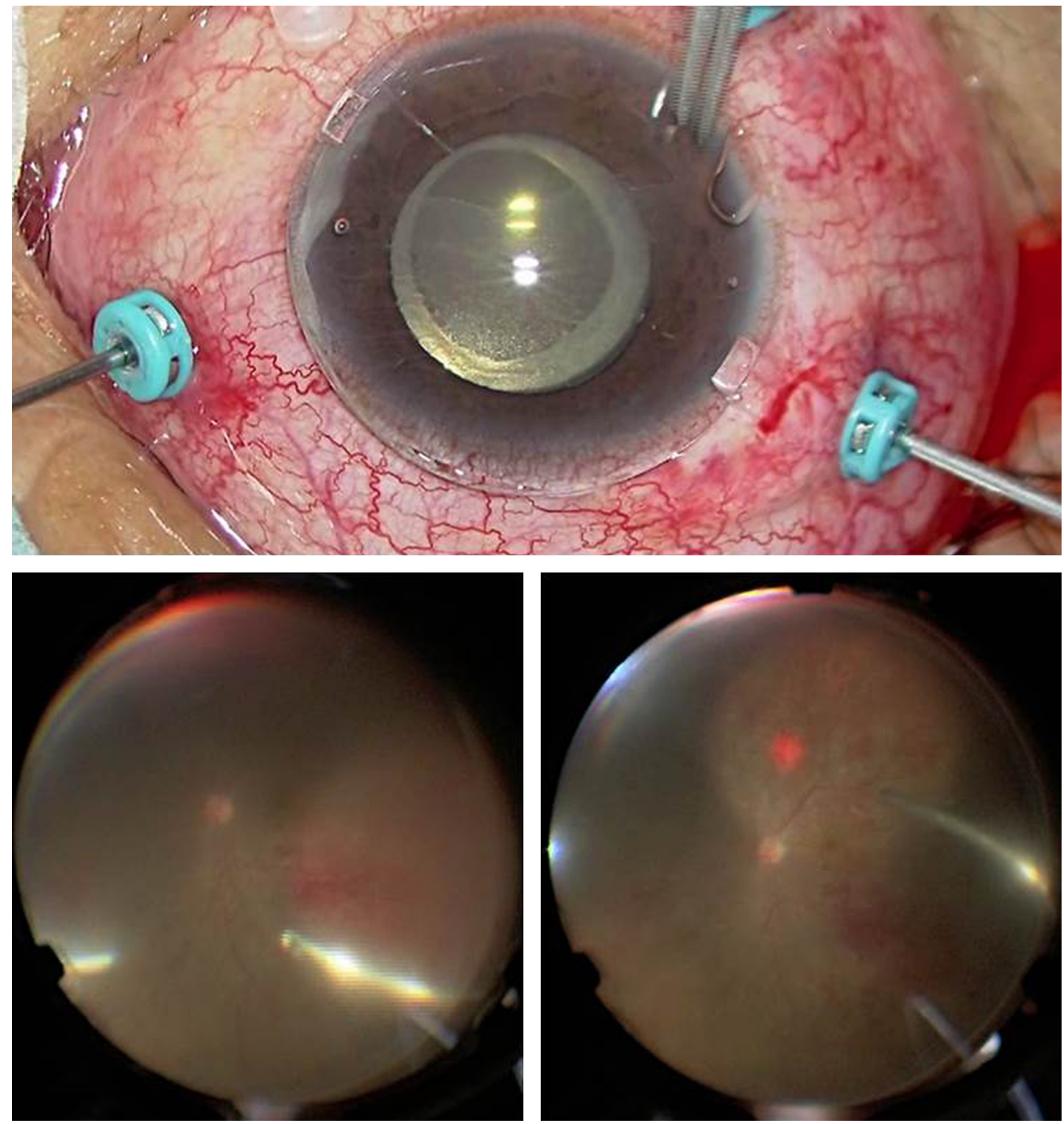

Figure 5 Application of the contact lens on the cornea in a case of diabetic retinopathy with corneal erosion and epithelial edema (top). Fundus view of the wide-angle viewing system alone (bottom left) and during use of the contact lens with the wide-angle viewing system (bottom right). 

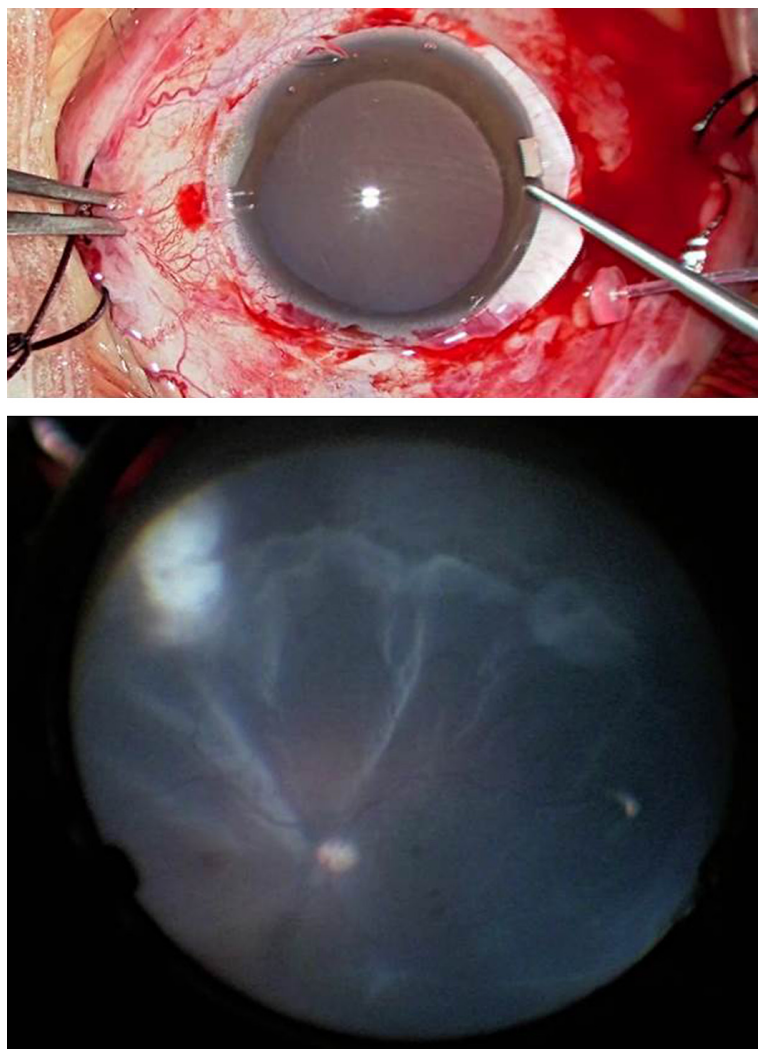

Figure 6 Application of the contact lens in a scleral buckling case (top). Fundus view through the contact lens with the wide-angle viewing system (bottom).

contact lens for myopia has also been reported to be ideal, it is designed to be movable on the cornea in order to prevent hypoxic damage during daily use. Therefore, this would be a disadvantage when performing vitrectomy. Ohno and Inoue ${ }^{8}$ developed a new antidrying contact lens that was made of quartz and had a diameter of $11.2 \mathrm{~mm}$ and a thickness of $1.0 \mathrm{~mm}$. Because of the thickness and weight, this smaller diameter lens tended to exhibit poor centering, in addition to requiring the use of a fixation system. In order to overcome these problems, this newly commercialized lens has a much larger outer diameter, is very thin, and has lightweight characteristics due to the use of polymethyl methacrylate. All of these contribute to the overall stabilization of the lens without having to use a fixation ring. ${ }^{4}$

Since viscoelastic material tends to easily dry, this can result in an irregular surface near the limbus that makes it difficult to observe the peripheral lesions. However, the large diameter of the contact lens makes it possible to provide a high quality of vision during surgeries at both the posterior pole and the peripheral area. Furthermore, this lens is designed to have a refractive power of zero, which prevents any disadvantageous changes in the panoramic view. Theoretically, the use of this lens can provide a slightly wider field of intraoperative view. ${ }^{4}$ Thus, the characteristics of this lens appear to be advantageous, especially in surgeries for rhegmatogenous retinal detachment or proliferative vitreoretinopathy when using both vitrectomy and scleral buckling.

Application of a magnifying contact lens is usually necessary in order to achieve peeling of the epimacular membrane or inner limiting membrane instead of a noncontact wide-angle viewing system. This lens is able to provide a good three-dimensional vision quality for the removal of proliferative membrane in diabetic retinopathy when using not only the 128-diopter but also the 60-diopter front lens of the Resight ${ }^{\circledR}$ fundus viewing system. This lens might be also useful for removing the epimacular membrane or the inner limiting membrane as compared to using a magnifying lens.

The use of this lens can improve the visualization of the surgical field in eyes with poor corneal status without abrasion of the corneal epithelium, which is definitely an advantage, especially in diabetic eyes.

In summary, this new commercially available lens for observing the fundus appears to be promising when used in combination with a noncontact wide-angle viewing system for both the vitrectomy and scleral buckling procedures.

\section{Disclosure}

The authors have no patent or license relevant to this lens. This work was partially supported by a research grant from HOYA Cooperation, Tokyo, Japan, which had no control over the interpretation, writing, or publication of this work. The authors report no other conflicts of interest in this work.

\section{References}

1. Aras C, Ucar D, Koytak A, Yetik H. Scleral Buckling with a non-contact wide-angle viewing system. Ophthalmologica. 2012;227:107-110.

2. Kita M, Fujii F, Kawagoe N, Hama S. Scleral buckling with a noncontact wide-angle viewing system in the management of retinal detachment with undetected retinal break: a case report. Clin Ophthalmol. 2013;7: 587-589.

3. Shiba K, Shiba T, Kodaka R, Kamizuru M, Shiba K. Retinal reattachment surgery using wide-angle viewing systems and chandelier illumination. J Ophthalmic Surg. 2010;23:301-304.

4. Chihara T, Kita M. New type of antidrying lens for vitreous surgery with a noncontact wide-angle viewing system. Clin Ophthalmol. 2013;7: 353-355.

5. Ohji M, Tada E, Futamura H. Combining a contact lens and wide-angle viewing system for a wider fundus view. Retina. 2011;31:1958-1960.

6. Ohno H. Combined use of high-reflective index vitrectomy meniscus contact lens and a noncontact wide-angle viewing system in vitreous surgery. Clin Ophthalmol. 2011;5:1109-1111.

7. Kamei M, Matsumura N, Sakaguchi H, Oshima Y, Ikuno Y, Nishida K. Commercially available rigid gas-permeable contact lens for protecting the cornea from drying during vitrectomy with a wide viewing system. Clin Ophthalmol. 2012;6:1321-1324.

8. Ohno H, Inoue K. An antidrying corneal contact lens for a noncontact wide-angle viewing system. Retina. 2011;31:1435-1436. 


\section{Publish your work in this journal}

Clinical Ophthalmology is an international, peer-reviewed journal covering all subspecialties within ophthalmology. Key topics include: Optometry; Visual science; Pharmacology and drug therapy in eye diseases; Basic Sciences; Primary and Secondary eye care; Patient Safety and Quality of Care Improvements. This journal is indexed on

Submit your manuscript here: http://www.dovepress.com/clinical-ophthalmology-journal
PubMed Central and CAS, and is the official journal of The Society of Clinical Ophthalmology (SCO). The manuscript management system is completely online and includes a very quick and fair peer-review system, which is all easy to use. Visit http://www.dovepress.com/ testimonials.php to read real quotes from published authors. 